\title{
Terrestrische Cyanobakterien als Quelle für antimikrobielle Wirkstoffe
}

\author{
MARCO WITTHOHN ${ }^{1}$, ANNA SCHWARZ ${ }^{1}$, DORINA STRIETH ${ }^{2}$, SELINA LENZ ${ }^{2}$, \\ ROLAND ULBER ${ }^{2}$, KAI MUFFLER ${ }^{1}$ \\ 1 FACHBEREICH 1 - LIFE SCIENCES AND ENGINEERING, TH BINGEN \\ 2 LEHRGEBIET BIOVERFAHRENSTECHNIK, TU KAISERSLAUTERN
}

\section{Cyanobacteria developed an enormous reservoir of bioactive secondary metabolites in order to prevail against competitive microorganisms and harsh environmental impacts. Many cyanobacterial substances with vast economical, medical and biotechnological potential have been described in the past. However, most of the examined bacteria are aquatic strains. We want to take a closer look on their terrestrial rela- tives which also possess a rich secondary metabolome that is still to explore.}

DOI: $10.1007 / \mathrm{s} 12268-020-1484-1$

(C) Die Autoren 2020

Cyanobakterien gehören zu den ältesten Organismen unseres Planeten, fossilen Funden zufolge entstanden sie vor etwa 3,5 Milliarden Jahren [1]. Als oxygen phototrophe Organismen haben sie einen großen Beitrag zur Entstehung der sauerstoffreichen Erdatmosphäre geleistet. Somit können Cyanobakterien als Motor für diesen folgenschweren und richtungsweisenden Evolutionsprozess angesehen werden.

\section{Hochwertige Syntheseprodukte}

Cyanobakterien sind sehr anpassungsfähige und robuste Bakterien, die in fast allen Lebensräumen vorkommen. Isolate wurden in Salz- und Süßwasser, Wüsten, Gletschern und heißen Quellen gefunden. Als Folge dieser Anpassungen produzieren Cyanobakterien eine Vielzahl hochwertiger Substanzen mit enormem kommerziellen Potenzial. Cyanobakterien sind im Gegensatz zu Pflanzen dazu in der Lage, Licht der Wellenlänge von 490-620 Nanometer photosynthetisch zu nutzen. Dafür haben sich spezielle Chromophore entwickelt, die Phycobiline. Auch verschiedene Carotinoide tragen zur Erweiterung des nutzbaren Lichtspektrums in Cyanobakterien bei. Beide Stoffklassen werden seit einiger Zeit erfolgreich in der Lebensmittelindustrie eingesetzt [2]. Gleichzeitig haben sich bei Cyanobakterien viele unterschiedliche Synthesewege für Energiespeicher entwickelt, was in einer großen Fülle unterschiedlicher Speicherstoffe resultiert. Darunter befinden sich vor allem langkettige ungesättigte Fettsäuren, wie z. B. Gamma-Linolensäure, Arachidonsäure, oder Docosahexaensäure, die aufgrund ihrer gesundheitsfördernden Wirkung ein gefragter Nahrungsmittelzusatz sind [3]. Des Weiteren produzieren Cyanobakterien eine Vielzahl biotechnologisch relevanter Substanzen, wie z. B. Lipide, Polysaccharide, Aminosäuren, Vitamine, Sterole und Enzyme. Unter den von Cyanobakterien produzierten Naturstoffen befinden sich häufig Toxine, wie von den alljährlich auftretenden „Cyanobakterien-Blüten“ in heimischen Badeseen bekannt ist. Das von Anabaena produzierte Anatoxin A sowie von Microcystis gebildete Microcystine gehören zu den stärksten natürlich vorkommenden Giften und können auch bei Menschen schwere gesundheitliche Schäden zur Folge haben. Weitaus nützlicher sind die vielzähligen cyanobakteriellen Sekundärmetabolite, bei denen unter anderem antimikrobielle oder antitumorale Wirkungen nachgewiesen werden konnten. Interessant ist dabei vor allem die Diversität der unterschiedlichen Substanzklassen. Diese reichen über Peptide, Polyketide, Alkaloide und Isoprenoide, bis hin zu antiviralen Polysacchariden.

\section{Besonderheit terrestrischer Cyanobakterien}

Bisher wurden vor allem aquatische Cyanobakterien als reiche Quelle für bioaktive Naturstoffe beschrieben. Doch auch terrestrische Cyanobakterien produzieren eine Vielzahl medizinisch und biotechnologisch interessanter Substanzen. Innerhalb der Ordnungen Chroococcales, Oscillatoriales, Pseudoanabaenales und Nostocales kommen neben aquatischen auch terrestrisch lebende Cyanobakterien vor [4]. Es ist bereits länger bekannt, dass Arten der Ordnungen Chroococcales, Oscillatoriales und Nostocales die meisten bioaktiven Sekundärmetabolite produzieren [3]. Darunter befinden sich vor allem Toxine, antivirale, antimykotische und antibakterielle Substanzen, aber auch Stoffe mit nachgewiesener Wirkung gegen Krebszellen, höhere Pflanzen sowie Algen. Neben einem breiten Spektrum an potenziell interessanten bioaktiven Sekundärmetaboliten haben terrestrische Cyanobakterien noch einige biotechnologische Vorteile gegenüber den aquatischen Arten zu bieten. Trotz ihrer natürlichen Lebensweise an Land können die meisten terrestrischen Cyanobakterien submers in (Photo-) Bioreaktoren kultiviert werden. Dabei weisen sie eine hohe Toleranz gegenüber schwankenden Kultivierungsbedingungen auf, wie Temperatur, Lichteintrag und Begasung [4]. Selbst eine komplette Austrocknung überstehen diese Organismen unbeschadet, was ganz neue Möglichkeiten im Bereich der Bioreaktortechnik ermöglicht [5]. Terrestrische Cyanobakterien sind also zum einen eine vielversprechende Quelle noch unbekannter bioaktiver Substanzen und besitzen zum anderen einzigartige physiologische Eigenschaften, die sie höchst interessant für biotechnologische Prozesse machen. Dieses Potenzial soll im Forschungscluster $i$ Process weiter ausgeschöpft werden.

\section{iProcess: intelligente Prozess-} entwicklung - von der Modellierung bis zum Produkt

Das Land Rheinland-Pfalz intensiviert seit 2018 über das Instrument der Forschungs- 


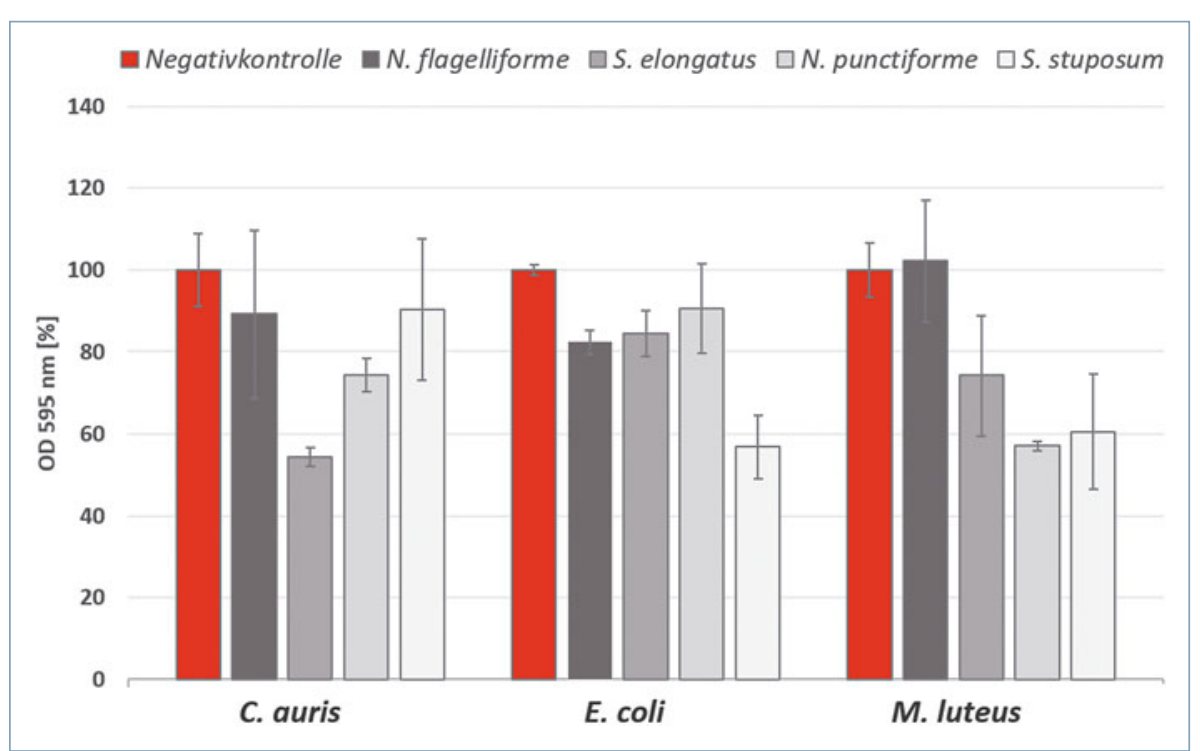

$\Delta$ Abb. 1: Erreichte Maximal-OD ${ }_{595 \mathrm{~nm}}$ der Indikatororganismen Candida auris, Escherichia coli und Micrococcus luteus nach Zugabe von Kulturüberständen der terrestrischen Cyanobakterien Nostoc flagelliforme, Synechococcus elongatus, Nostoc punctiforme und Scytonema stuposum (1:1). Alle Cyanobakterien wurden als Flüssigkultur phototroph $\left(30 \mu \mathrm{mol} \mathrm{m}{ }^{-2} \mathrm{~s}^{-1}\right)$ bei $27{ }^{\circ} \mathrm{C}$ und 120 rpm in BG-11-Medium kultiviert. Proben wurden nach 2, 5 und 7 Tagen entnommen. Dargestellt sind die Daten der Proben mit höchster Inhibitionswirkung. Rot: Negativkontrolle mit BG-11-Medium. Fehlerbalken zeigen Standardabweichung, $n=4$. kollegs die wissenschaftliche Zusammenarbeit von Hochschulen und Universitäten, wobei innerhalb von Verbundprojekten die Durchführung kooperativer Promotionen der wissenschaftlichen Mitarbeiter/innen der beteiligten Hochschulen ermöglicht wird. Zu den ersten geförderten Verbünden zählt das interdisziplinäre Forschungskolleg „iProcess“ (https://www.mv.uni-kl.de/iprocess), in dem sich Arbeitsgruppen der HS Trier, der TH Bingen und der TU Kaiserslautern vernetzt haben, um die verfahrenstechnischen Grundlagen zu erarbeiten, damit Pilze und Cyanobakterien als Produktionsorganismen für pharmazeutisch wirkende Substanzen genutzt werden können.

\section{Screening ausgewählter terrestrischer Cyanobakterien nach geeigneten Substanzen}

Ein Teilbereich von $i$ Process beschäftigt sich mit der Produktion antimikrobieller Wirkstoffe mittels terrestrischer Cyanobakterien. Dabei soll der gesamte Prozess entwickelt werden - von der Modellierung über den Upstream-Bereich und die Entwicklung geeigneter Reaktoren bis hin zum Downstream-Prozess.

Für die Entwicklung eines Produktionsprozesses muss zunächst eine geeignete Zielsubstanz gefunden werden. Dafür wurden cyanobakterielle Kulturüberstände auf anti- biotische Wirkung getestet und vorhandene Genomsequenzen mittels der Biosynthesecluster-Analyse-Software antiSMASH auf Gencluster mit eventuell bioaktiven Genprodukten hin überprüft [6]. Die Produktion antimikrobieller Sekundärmetabolite wurde mittels Wachstumstests in Mikrotiterplatten untersucht, in denen die Indikatororganismen Escherichia coli, Micrococcus luteus und Candida auris mit dem jeweiligen Cyanobakterien-Kulturüberstand versetzt wurden (Abb. 1). Durch die Wahl eines Gram-negativen und eines Gram-positiven Bakteriums sowie einer Hefe als Testorganismen kann ein relativ breites Wirkspektrum abgedeckt werden.

Die Kulturüberstände von S. elongatus, $N$. punctiforme und S. stuposum zeigen die deutlichsten Hemmwirkungen. Das Wachstum der Hefe $C$. auris wurde durch ins Medium sekretierte Substanzen von S. elongatus um fast 50 Prozent inhibiert, jenes der Bakterien besonders durch Kulturüberstände von $N$. punctiforme und S. stuposum. Dabei hemmten von $N$. punctiforme gebildete Stoffe vor allem das Gram-positive Bakterium M. luteus. Kulturüberstände von S. stuposum wirkten sich auf das Wachstum beider Bakterien negativ aus.

Die antiSMASH-Analyse konnte einige putativ von den ausgewählten terrestrischen Cyanobakterien produzierte Substanzen aufzeigen, welche die in Abbildung 1 gezeigten Hemmwirkungen auslösen könnten (Tab. 1).

In den Cyanobakterien-Genomen wurden potenzielle Biosynthesecluster für Substanzen gefunden, die eine antibiotische Wirkung aufweisen könnten. Dabei ist es ebenfalls möglich, dass als cytotoxisch eingestufte Stoffe bisher noch nicht auf ihre Wirksamkeit gegen Mikroorganismen getestet wurden und demnach nicht als Antibiotika beschrieben sind. Für viele von antiSMASH gefundene Cluster konnte kein putatives Genprodukt gefunden werden, demnach können die Metabolome der untersuchten Cyanobakterien noch eine Vielzahl unentdeckter Substanzen unterschiedlicher Stoffklassen und Bioaktivität enthalten.

Die gezeigten Ergebnisse deuten bereits an, dass terrestrische Cyanobakterien großes Potenzial für eine ganze Bandbreite wertvoller Sekundärmetabolite bergen, die nicht zuletzt im Kampf gegen Viren und multi-
Tab. 1: Ergebnisse der antiSMASH-Analyse, beispielhaft für zwei der genutzten CyanobakterienStämme. Aufgeführt ist eine Auswahl bioaktiver Stoffe mit putativ antibiotischer Wirkung. Diese wurden durch die Ähnlichkeit einzelner Gene innerhalb der cyanobakteriellen Genom-Sequenz zu Genen bereits bekannter Biosynthesecluster in anderen Organismen ermittelt. NRPS: nicht-ribosomale Peptidsynthetase; PKS: Polyketidsynthase.

\begin{tabular}{|c|c|c|c|c|}
\hline Organismus & Biosynthesecluster & $\begin{array}{l}\text { Putatives Genprodukt } \\
\text { Übereinstimmung [\%] }\end{array}$ & Stoffklasse & Bioaktivität \\
\hline \multirow[t]{2}{*}{ N. flagelliforme } & NRPS/PKS & Nostophycin (27) & Cycl. Peptid & cytotoxisch [7] \\
\hline & PKS & Minutissamid A/C/D (46) & Cycl. Depsipeptid & $\begin{array}{l}\text { antiproliferativ, } \\
\text { cytotoxisch [8] }\end{array}$ \\
\hline \multirow[t]{4}{*}{ N. punctiforme } & NRPS-like/PKS & $\begin{array}{l}\text { Merocyclophane C/D } \\
\text { (33) }\end{array}$ & Polyketid & cytotoxisch [9] \\
\hline & NRPS/PKS/Bacteriocin & Nostophycin (27) & Cycl. Peptid & cytotoxisch [7] \\
\hline & PKS & Minutissamid A/C/D (46) & Cycl. Depsipeptid & $\begin{array}{l}\text { antiproliferativ, } \\
\text { cytotoxisch [8] }\end{array}$ \\
\hline & NRPS/PKS & Cyanopeptolin (75) & Peptid & cytotoxisch [10] \\
\hline
\end{tabular}


resistente Keime den entscheidenden Beitrag leisten könnten.

\section{Danksagung}

Dieses Projekt ist Teil des Forschungsclusters „iProcess“ und wird finanziell vom Land Rheinland-Pfalz unterstützt. Wir danken Prof. Dr. Burkhard Büdel (Abt. Pflanzenökologie und Systematik, TU Kaiserslautern) für die freundliche Bereitstellung der Cyanobakterien. Wir danken ebenfalls der DFG für die finanzielle Unterstützung der Projekte MU 2985/3-1 und UL 170/16-1.

\section{Literatur}

[1] Farrokh P, Sheikhpour M, Bavandi R (2019) Cyanobacteria as an eco-friendly resource for biofuel production : A critical review. Biotechnol Prog 35:1-16

[2] Borowitzka MA (1995) Microalgae as sources of pharmaceuticals and other biologically active compounds. J Appl Phycol 7:3-15

[3] Burja AM, Banaigs B, Abou-Mansour E et al. (2007) Marine cyanobacteria - a prolific source of natural products. Tetrahedron Lett 57:9347-9377

[4] Lakatos M, Strieth D (2008) Terrestrial microalgae: novel concepts for biotechnology and applications. In: Cánovas FM, Lüttge U, Matyssek R (Hrsg.) Progress in botany. Springer International Publishing, Basel, 269-312
[5] Muffler K, Lakatos M, Schlegel C et al. (2014) Application of biofilm bioreactors in white biotechnology BT In: Muffler K, Ulber R (Hrsg.) Productive biofilms. Springer International Publishing, Basel, 123-161

[6] Medema MH, Blin K, Cimermancic P et al. (2011)

AntiSMASH: rapid identification, annotation and analysis of secondary metabolite biosynthesis gene clusters in bacterial and fungal genome sequences. Nucleic Acids Res 39:339-346 [7] Fujii K, Sivonen K, Kashiwagi T et al. (1999) Nostophycin, a novel cyclic peptide from the toxic Cyanobacterium Nostoc. J Org Chem 64:5777-5782

[8] Kang HS, Krunic A, Shen Q et al. (2011) Minutissamides A-D, antiproliferative cyclic decapeptides from the cultured cyanobacterium Anabaena minutissima. J Nat Prod 74:15971605

[9] May D, Luo S, Krunic A et al. (2015) Isolation and structure elucidation of merocyclophane $\mathrm{C}$ from the cultured cyanbacterium Nostoc sp. (UIC 10110). Planta Med 81:PT12

[10] Faltermann S, Zucchi S, Kohler E et al. (2014) Molecular effects of the cyanobacterial toxin cyanopeptolin (CP1020) occurring in algal blooms: Global transcriptome analysis in zebrafish embryos. Aquat Toxicol 149:33-39

Funding note: Open Access funding enabled and organized by Projekt DEAL.

Open Access: Dieser Artikel wird unter der Creative Commons Namensnennung 4.0 International Lizenzveröffentlicht, welche die Nutzung, Vervielfältigung erlaubt, sofern Sie den/die ursprünglichen Autor(en) und die Quelle ordnungsgemäß nennen, einen Link zur Creative Commons Lizenz beifügen und angeben, ob Anderungen vorgenommen wurden. Die in diesem Artikel enthaltenen Bilder und sonstiges Drittmaterial unterliegen ebenfalls der genannten Creative Commons Lizenz, sofern sich aus der Abbildungslegen nichts anderes ergibt. Sofern das betreffende Material nicht unter der enannten Creative Commons Lizenz steht und die betreffende Handlung nicht nach gesetzlichen Vorschriften erlaubt ist, ist für die oben aufgefüh Rechteinhabers einz des Materials die Eirils zur Lizenzinformation auf http://creativecommons.org/licenses/by/4.0/deed.de.

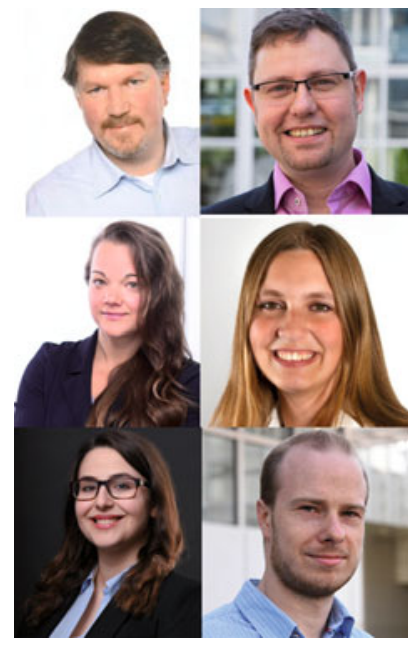

Roland Ulber, Dorina Strieth, Selina Lenz (links) Kai Muffler, Anna Schwarz, Marco Witthohn (rechts)

Korrespondenzadresse:

Prof. Dr.-Ing. Kai Muffler

Fachbereich 1 - Life Sciences \& Engineering Technische Hochschule Bingen

D-55411 Bingen am Rhein

k.muffler@th-bingen.de

\section{Hier steht} eine Anzeige. 